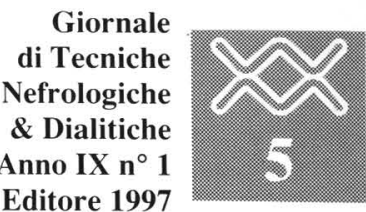

\title{
Insufficienza renale cronica e danno carotideo
}

\author{
L. Bonfante' ${ }^{1}$ A. Rossi' ${ }^{2}$ A. D’Angelo ${ }^{1 *}$
}

\section{${ }^{1}$ Divisione di Nefrologia I I, ${ }^{2}$ Patologia Medica I ${ }^{\text {a }}$ Università degli Studi di Padova, Padova}

\author{
* Hanno partecipato a questo studio: A. Antonello, L. Calò, A. Giacomini, M. Normanno, L. Maldini, M. Fusaro, V. Bordin, \\ C. Abaterusso, E. De Vivo (Divisione di Nefrologia Ia, Università degli Studi di Padova); A. Calabrò, M.L. Calabrò, D. Bianchi, \\ E. Abbruzzese (Patologia Medica Ia, Università degli Studi di Padova); R. Cavagna, L. De Silvestro (Servizio di Nefrologia e Dialisi, \\ Ospedale di Belluno); A. Bonadonna, S. Mastrosimone (Servizio di Nefrologia e Dialisi, Ospedale di Camposampiero)
}

a prima causa di morte nei pazienti nefropatici è dovuta a complicanze vascolari (1-3). L'ipertensione arteriosa, le alterazioni del metabolismo lipidico e della coagulazione, più frequenti nei soggetti con insufficienza renale cronica (IRC) che nella popolazione generale, potrebbero contribuire a spiegare questo fenomeno (4-15). Per questi motivi abbiamo valutato la prevalenza di lesioni aterosclerotiche delle carotidi extracraniche (LC) nei pazienti con IRC non nefrosica, non diabetica e l'associazione tra la gravità delle stenosi ed una serie di fattori di rischio.

\section{Pazienti}

Sono stati studiati 203 soggetti suddivisi in due gruppi: il I gruppo comprendeva 103 pazienti $(68 \mathrm{M}, 35 \mathrm{~F})$ affetti da IRC ed il gruppo II 100 soggetti di controllo (60M, 40F).

Nel gruppo di controllo l'azotemia, la creatininemia e l'esame urine sono risultati nella norma.

I criteri di esclusione, in entrambi i gruppi, comprendevano la presenza di diabete mellito e sintomi o storia clinica positivi per patologia cerebrovascolare.
Dal gruppo I sono stati esclusi i pazienti con proteinuria delle 24 ore superiore a 3 grammi e/o con insufficienza renale dovuta a vasculite o stenosi dell'arteria renale.

Le alterazioni lipidiche e proteiche riscontrate nella sindrome nefrosica risultano essere diverse da quelle presenti nell'IRC non nefrosica $(16,17)$. La sindrome nefrosica può comportare quindi un rischio diverso per l'aterosclerosi. Per tale motivo sono stati esclusi dal nostro studio i pazienti con sindrome nefrosica associata a IRC.

In entrambi i gruppi la percentuale di ipertesi $(84 \%)$ e normotesi $(16 \%)$ era identica. L'ipertensione è stata diagnosticata secondo i criteri stabiliti dall'OMS. La diagnosi, inoltre, è stata posta in caso di anamnesi positiva per ipertensione e/o per assunzione cronica di farmaci antiipertensivi.

L'eziologia dell'IRC nel gruppo I è stata determinata in 79 soggetti $(76.7 \%)$ sulla base di accertamenti clinici completi, comprendenti esami di laboratorio, indagini strumentali e, in casi selezionati, la biopsia renale. In 38 pazienti $(36.9 \%)$ la causa iniziale era una glomerulonefrite cronica, in $14(13.6 \%)$ la malattia renale policistica, in $11(10.6 \%)$ la nefrite tubu- lointerstiziale, in 7 (6.8\%) la nefroangiosclerosi ipertensiva, in 4 (3.9\%) la nefrolitiasi, in $3(2.9 \%)$ la tubercolosi renale, in $1(1 \%)$ il rene a spugna della midollare, nei restanti $24(23.3 \%)$ la causa è rimasta sconosciuta.

Nei pazienti di controllo ipertesi tutte le possibili cause di ipertensione secondaria sono state escluse sulla base di accurati accertamenti clinici e di laboratorio. In entrambi i gruppi sono stati valutati: il sesso, l'età, il BMI, la pressione arteriosa, l'abitudine al fumo, il fibrinogeno, il colesterolo totale(CT), i trigliceridi (TG), l'emocromo.

Sono stati considerati fumatori coloro che fumavano correntemente, non fumatori quelli che non avevano mai fumato o che avevano smesso da almeno 10 anni, ex fumatori i soggetti che avevano smesso di fumare in un periodo di tempo compreso tra 10 anni ed il mese precedente lo studio (18).

Per diagnosticare la presenza di cardiopatia ischemica tutti i pazienti di entrambi i gruppi sono stati sottoposti ad un'accurata valutazione clinica, hanno eseguito l'ECG e, in casi selezionati, il test da sforzo.

Per la diagnosi di arteriopatia periferica si sono utilizzati: anamnesi, esame obiet- 
tivo, e, nei casi in cui veniva ritenuto necessario, è stato eseguito l'esame doppler con calcolo dell'indice di Winsor. I pazienti del gruppo I sono stati suddivisi in tre sottogruppi (Tab. II):

1) Sottogruppo Ia: pazienti con IRC in terapia conservativa;

2) Sottogruppo Ib: pazienti con IRC in terapia sostitutiva;

3) Sottogruppo Ic: pazienti sottoposti a trapianto renale.

In tutti i pazienti del gruppo I è stato effettuato uno studio completo del profilo lipidico che comprendeva: LDL, HDL, Lp(a), ApoAI, ApoAII e ApoB.

\section{Metodi}

Per la determinazione delle HDL è stato utilizzato il metodo della precipitazione con composti polianionici delle lipoproteine contenenti ApoB (VLDL e LDL) (19).

Le ApoAI, le ApoAII e le ApoB sono state determinate con l'immunoelettroforesi utilizzando il nefelometro Behring e specifici anticorpi (Behring, L'Aquila, Italia, per ApoAI e ApoB; Daiichi Tokio, Giappone per ApoAII). Le LDL sono state ottenute con la formula di Friedewald (20) nei pazienti con livelli di TG più bassi di $250 \mathrm{mg} / \mathrm{dl}$.

Con il metodo immunoenzimatico ELISA si sono ottenuti i valori di $\mathrm{Lp}(\mathrm{a})$ (Terumo medical Corporation Elkton, MD, USA) (21).

Il fibrinogeno è stato dosato con metodo immunonefelometrico e anticorpi specifici (Behring, L'Aquila, Italia).

\section{Valutazione duplex}

Sono stati esaminati i seguenti vasi: carotide comune, biforcazione carotidea, tratto prossimale della carotide interna (22). E stato utilizzato un Eco-doppler (duplex) ad alta risoluzione, Biosound 2000 , dotato di sonda meccanica da $4 \mathrm{~cm}$ con frequenza di $8 \mathrm{Mhz}$. Questo sistema ha una risoluzione assiale e laterale di circa 0.385 e $0.5 \mathrm{~mm}$, rispettivamente, che consente di individuare con accuratezza anche lesioni parietali iniziali quali l'ispessimento intimo-mediale (23).

Il doppler pulsato, di cui è dotato lo strumento, permette, inoltre, di valutare le caratteristiche del flusso ematico. I dettagli di questa tecnica sono stati riportati in studi precedenti $(24,25)$.

Nel nostro dipartimento vascolare è stata validata la metodica con l'angiografia convenzionale dimostrando una buona correlazione. Abbiamo confrontato, infatti, i risultati del duplex su 340 arterie (dati non pubblicati) con le rispettive arteriografie. Le stenosi rilevate con le due metodiche (duplex ed angiografia) sono state classificate nei seguenti gruppi: $0 \%, 1-19 \%, 20-49 \%, 50-74 \%, 75-99 \%$, $100 \%$.

I risultati del duplex rientravano nello stesso gruppo di dati ottenuti con l'angiografia per le sei classi di stenosi nelle seguenti percentuali rispettivamente: $84 \%, 80 \%, 78 \%, 78 \%, 79 \%, 97 \%$. La riproducibilità nel nostro laboratorio, come dimostrato in un precedente lavoro (24) è ottima.

Tutti gli esami sono stati eseguiti dallo stesso operatore, che era all'oscuro delle indicazioni all'esame.

Le misurazioni sono state eseguite in telediastole.

Da un punto di vista qualitativo le placche carotidee possono essere classificate in 5 tipi:

1) Ispessimento intimo-mediale

2) Placca poco ecogena

3) Placca mista

4) Placca intermedia

5) Placca molto ecogena

secondo Felix modificato (22).

La misurazione dello spessore veniva eseguita solo sulla parete profonda dell'arteria meglio visualizzata con l'immagine B-Mode, rispetto alla parete superficiale. L'ispessimento intimo-mediale è definito come uno spessore maggiore di $1.2 \mathrm{~mm}$ tra l'interfaccia lume-intima e l'interfaccia media-avventizia, in accordo con i criteri accettati (26).

L'ispessimento intimo-mediale è stato misurato su tutti i distretti esplorabili: carotidi comuni, biforcazione carotidea (27), tratto prossimale dell'arteria carotide interna, bilateralmente.

Va tenuto presente che l'ultrasonografia B-mode ad alta risoluzione consente di riconoscere un ispessimento intimo-mediale, anche quando non c'è un'importante ostruzione del lume e/o un'alterazione del flusso $(28,29)$. Non si può, peraltro, con l'ecografia, distinguere tra loro le lesioni come la stria lipidica diffusa o l'ispessimento subendoteliale o l'ipertrofia mediale (26). Tuttavia, l'ispessimento intimo-mediale è un'alterazione della parete arteriosa e come tale va rilevato .
In base alla loro gravità le lesioni sono classificate in 5 classi come precedentemente indicato $(24,30)$.

La percentuale di stenosi è stata calcolata mediante la determinazione del diametro arterioso totale e del diametro residuo utilizzando il cursore elettronico di cui è dotato lo strumento; il diametro residuo è stato misurato nel punto di massima stenosi determinata dalla lesione, mentre il diametro totale è stato misurato nel tratto dell'arteria normale più prossimo alla lesione.

Queste misurazioni sono state effettuate nelle tre proiezioni longitudinali (anteriore, laterale, postero-laterale), facendo, poi, la media delle misurazioni.

Le stenosi senza variazioni di flusso sono state definite come classe 1 e 2 , in accordo con la loro gravità (1-19\% e 20$49 \%$ ); le stenosi con alterazioni di flusso come classe 3, 4 e 5 (50-74\%; 75-99\% e $100 \%$ rispettivamente).

È risaputo comunque, che, come tutte le classificazioni, questi dati sono indicatori approssimativi di gravità perché molti fattori possono influenzare la rilevanza clinica ed emodinamica delle stenosi (31).

Per eseguire l'analisi multivariata, la media aritmetica della percentuale delle stenosi più gravi dei due lati, presa come indice di gravità, è stata utilizzata come variabile dipendente. Per lo studio di prevalenza è stata esaminata la presenza o l'assenza di lesioni a livello di carotide comune, biforcazione ed origine di carotide interna dei due lati.

\section{Analisi statistica}

I risultati ottenuti sono stati espressi come media \pm deviazione standard. Per il confronto fra i gruppi è stata utilizzata l'analisi della varianza seguita dal metodo di Scheffè per comparazioni multiple. Per il confronto delle percentuali è stato utilizzato il test Chi quadro $(32,33)$; per valutare l'effetto sulla gravità delle lesioni carotidee (LC) e dell'IRC e dei fattori di rischio, come variabili di gruppo e covariate, rispettivamente, è stata, inoltre, eseguita l'analisi multivariata della varianza. Il metodo blackward è stato usato per valutare l'interazione tra le variabili, usando una probabilità di F-to rimove (pout) di 0.10 (34). Tutte le analisi sono state eseguite con programma statistico SPSS (3.0; SPSS inc, Chicago). 


\section{Risultati}

\section{Caratteristiche cliniche dei pazienti}

Nella Tabella I abbiamo riportato le caratteristiche cliniche dei pazienti dei due gruppi comprendenti: età, sesso, BMI, percentuale di ipertesi, colesterolo totale, trigliceridi, fibrinogeno e creatinina.

I due gruppi sono risultati simili per presenza, durata dell'ipertensione ed uso di farmaci antiipertensivi.

In entrambi i gruppi, la percentuale di fumatori, di non fumatori e di ex-fumatori non presentava differenze significative.

I livelli di trigliceridi e di fibrinogeno sono risultati significativamente più alti nei pazienti uremici rispetto ai controlli $(\mathrm{p}<$ $0.01)$, mentre il BMI è stato significativamente maggiore nei controlli rispetto al gruppo di pazienti con IRC ( $\mathrm{p}<0.001)$.

$\mathrm{Vi}$ erano 23 pazienti con coronaropatia nel gruppo I e 7 nel gruppo di controllo $(\mathrm{p}=0.04)$. Cinque soggetti presentavano arteriopatia periferica nel gruppo I e 6 nel gruppo II $(\mathrm{p}=\mathrm{ns})$.

In Tabella II, abbiamo riportato le caratteristiche cliniche dei 3 sottogruppi di pazienti uremici. Il BMI e la pressione arteriosa media sono risultati più alti nel sottogruppo Ia rispetto al sottogruppo Ib $(\mathrm{p}<0.05)$.

Il sottogruppo Ic presentava livelli di colesterolo, trigliceridi, HDL-colesterolo, ApoAI e ApoAII, maggiore rispetto al sottogruppo Ia $(p<0.05)$. Inoltre nel sottogruppo Ic si registrava un aumento di colesterolo totale rispetto al gruppo $\mathrm{Ib}$ $(\mathrm{p}<0.05)$.

Nella Tabella III sono state riportate le caratteristiche cliniche dei pazienti normotesi del gruppo I e II. I due gruppi differivano significativamente solo per i valori di creatininemia e di fibrinogeno che sono risultati maggiori nei pazienti del gruppo I $(\mathrm{p}<0.0001, \mathrm{p}<0.01)$.

\section{Lesioni carotidee al duplex}

Dei 203 soggetti esaminati, 2 pazienti appartenenti al gruppo I sono stati esclusi per la scarsa qualità dell'esame duplex. La prevalenza di lesioni carotidee (LC)

TABELLA I - CARATTERISTICHE DEI PAZIENTI CON INSUFFICIENZA RENALE CRONICA (GRUPPO I) E DEI SOGGETTI DI CONTROLLO (GRUPPO II)

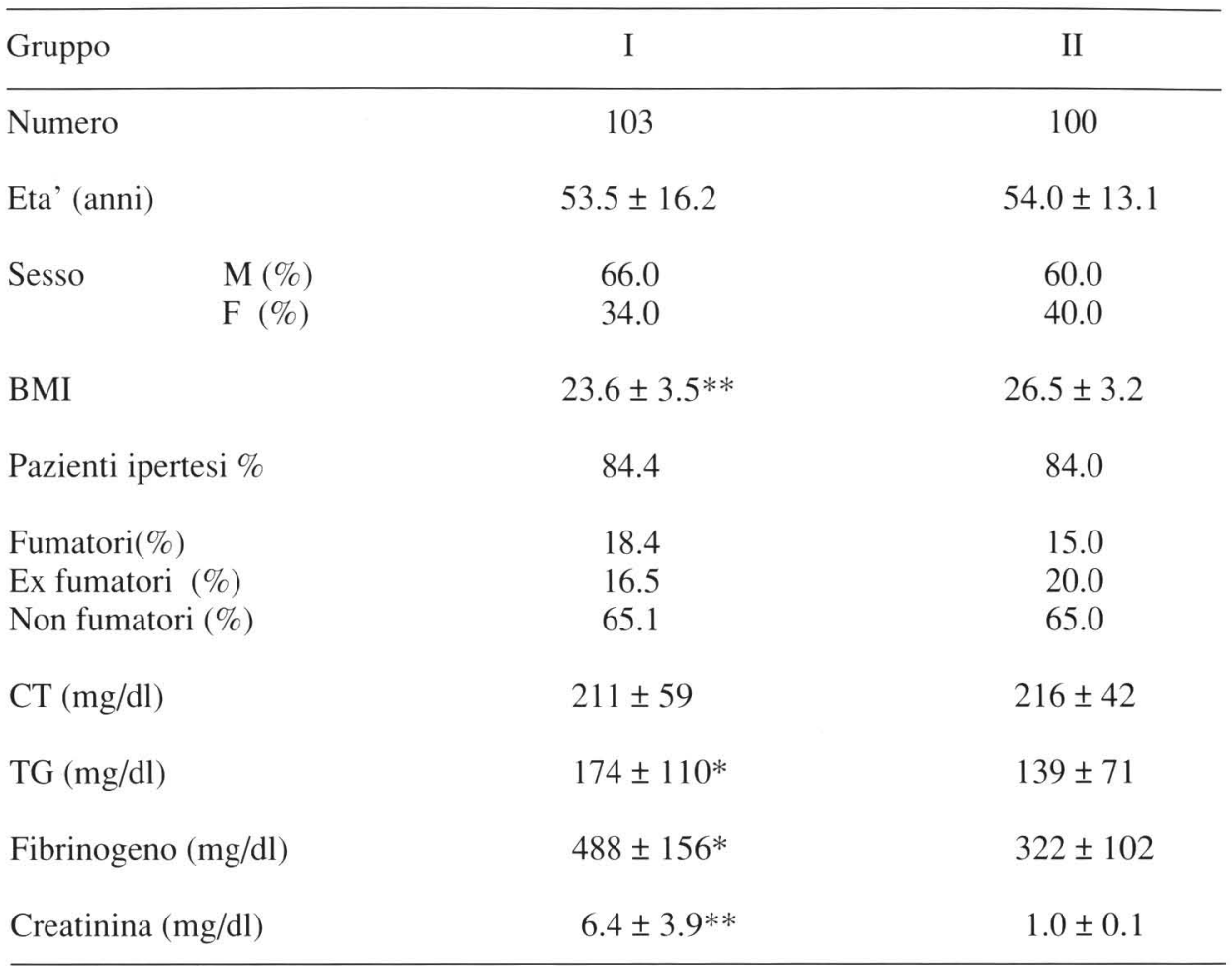

Valori con più e meno sono Media \pm Deviazione Standard

BMI: Indice di massa corporea;

CT: Colesterolo Totale; TG: Trigliceridi.

$* \mathrm{p}<0.01$

$* * \mathrm{p}<0.001$

fu rispettivamente di $62.4 \%(\mathrm{M}=$ $67.6 \%$ e $\mathrm{F}=51.5 \%$ ) nei pazienti uremici e $47.0 \%(\mathrm{M}=52.5 \%$ e $\mathrm{F}=35.9 \%)$ nel gruppo di controllo $(\mathrm{p}=0.04)$ (Fig. 1).

Il $68.3 \%$ dei casi del gruppo I ed il $61.7 \%$ del gruppo II presentavano LC bilateralmente, mentre il riscontro di LC monolateralmente è stato del $31.7 \%$ dei casi nel gruppo I e $38.3 \%$ nel gruppo II ( $\mathrm{p}=\mathrm{ns}$ ).

Tra i pazienti ipertesi del gruppo I la prevalenza di LC è stata del $62.4 \%$ rispetto al 52.6\% del gruppo di ipertesi di controllo (Fig. 2, p = ns). Nei pazienti normotesi il riscontro di LC è stato di $62.5 \%$ nel gruppo I contro il $18.7 \%$ nel gruppo II (Fig. 2, p = 0.03).

L' analisi multivariata della varianza ha dimostrato che la gravità delle LC aggiustata per le covariabili (fattori di rischio) era spiegata dall'appartenenza al gruppo IRC ed al sesso maschile, $(\mathrm{F}=9.25, \mathrm{p}=$ $0.002)$ e $(\mathrm{F}=10.89 ; \mathrm{p}=0.001)$.
La coronaropatia si associava nell' $86 \%$ dei casi in entrambi i gruppi a LC, mentre l'arteriopatia periferica era associata alle LC nel $100 \%$ dei casi del gruppo I e nel $67 \%$ nei controlli.

La Tabella IV dimostra la frequenza dell'ispessimento intimo-mediale e delle placche aterosclerotiche.

Sono stati riscontrati 37 ispessimenti intimo-mediali $(33.0 \%$ del totale delle lesioni) nel gruppo I e 34 (45.3\%) nel gruppo II, mentre le placche aterosclerotiche determinanti stenosi di vario grado sono state osservate in 69 pazienti con IRC $(67.0 \%$ delle lesioni totali) e in 41 nei controlli (54.7\%).

Non si sono registrate, inoltre, differenze significative fra i due gruppi riguardo il tipo di lesioni o le caratteristiche di superficie.

La prevalenza delle lesioni nei tre sottogruppi di pazienti affetti da IRC è stata di $61.8 \%$ in Ia; $67.7 \%$ in $\mathrm{Ib}$ e $53.3 \%$ in Ic (Fig. 3). 
Effetti dei fattori di rischio sulla gravità delle $\mathrm{LC}$

L'analisi multivariata eseguita sui pazienti con IRC, introducendo come va- riabile dipendente il grado di stenosi ha rivelato una correlazione positiva con età ( $p=0.001)$, numero di globuli bianchi $(\mathrm{p}=0.02)$, trigliceridi $(\mathrm{p}=0.02)$ e fibrinogeno $(p=0.09)$.

Non sono state evidenziate correlazioni significative con i livelli di pressione ar-

TABELLA II - CARATTERISTICHE DEI SOTTOGRUPPI DI PAZIENTI CON INSUFFICIENZA RENALE

\begin{tabular}{|c|c|c|c|}
\hline 'Sottogruppi & I a & $\mathrm{Ib}$ & I c \\
\hline Numero & 55 & 33 & 15 \\
\hline Età (anni) & $54.6 \pm 16.7$ & $57.2 \pm 13.7$ & $40.9 \pm 14.2$ \\
\hline $\begin{array}{l}\mathrm{M}(\%) \\
\mathrm{F}(\%)\end{array}$ & $\begin{array}{l}63.6 \\
36.4\end{array}$ & $\begin{array}{l}69.7 \\
30.3\end{array}$ & $\begin{array}{l}66.6 \\
33.4\end{array}$ \\
\hline BMI & $23.4 \pm 3.6^{*}$ & $22.2 \pm 3.0$ & $23.8 \pm 2.7$ \\
\hline Pazienti ipertesi (\%) & 85.4 & 81.8 & 86.6 \\
\hline $\begin{array}{l}\text { Fumatori }(\%) \\
\text { Ex fumatori }(\%) \\
\text { Non fumatori }(\%)\end{array}$ & $\begin{array}{l}21.8 \\
23.6 \\
54.6\end{array}$ & $\begin{array}{c}48.4 \\
6.1 \\
45.5\end{array}$ & $\begin{array}{c}6.6 \\
13.3 \\
80.1\end{array}$ \\
\hline $\mathrm{CT}(\mathrm{mg} / \mathrm{dl})$ & $203 \pm 49 \dagger$ & $200 \pm 42^{\circ}$ & $274 \pm 91$ \\
\hline $\mathrm{TG}(\mathrm{mg} / \mathrm{dl})$ & $161 \pm 85 \dagger$ & $168 \pm 104$ & $249 \pm 184$ \\
\hline $\mathrm{HDL}(\mathrm{mg} / \mathrm{dl})$ & $40.5 \pm 13.7 \dagger$ & $43.1 \pm 11.3$ & $50.9 \pm 19.8$ \\
\hline LDL (mg/dl) & $132 \pm 40$ & $123 \pm 32$ & $144 \pm 11$ \\
\hline APO-AI (mg/dl) & $136 \pm 26 \dagger$ & $141 \pm 19$ & $155 \pm 31$ \\
\hline APO-AII (mg/dl) & $21.3 \pm 6.5 \dagger$ & $23.8 \pm 4.0$ & $26.8 \pm 6.5$ \\
\hline $\begin{array}{l}\mathrm{Lp}(\mathrm{a})(\mathrm{mg} / \mathrm{dl}) \\
\text { Mediana (range) }\end{array}$ & $18.1(1.8-100.0)$ & $9.2(0.5-67.9)$ & $24.5(2.8-70.0)$ \\
\hline $\begin{array}{l}\log \mathrm{Lp}(\mathrm{a}) \\
\text { Mediana (range) }\end{array}$ & $1.2(0.3-2.0)$ & $1.0(0.1-1.8)$ & $1.4(0.4-1.8)$ \\
\hline Creatinina (mg/dl) & $5.3 \pm 3.3^{* \dagger}$ & $10.5 \pm 2.0^{\circ}$ & $1.8 \pm 0.7$ \\
\hline Fibrinogeno (mg/dl) & $485 \pm 150$ & $507 \pm 186$ & $451 \pm 70$ \\
\hline
\end{tabular}

Insufficienza renale cronica in terapia conservativa (Ia), in terapia sostitutiva (Ib), dopo trapianto renale (Ic)

Valori con più e meno sono Media \pm Deviazione Standard

BMI: Indice di Massa Corporea; CT: colesterolo totale;

TG: Trigliceridi; HDL: HDL colesterolo;

LDL: LDL colesterolo; Apo-AI: Apoproteina AI;

Apo-AII: Apoproteina A II; LP(a): Lipoproteina a.

$* \mathrm{p}<0.05$ gruppo Ia contro gruppo Ib

$\dagger \mathrm{p}<0.05$ gruppo Ia contro gruppo Ic

${ }^{\circ} \mathrm{p}<0.05$ gruppo Ib contro gruppo Ic

Le complicanze vascolari, come l'infarto del miocardio e l'ictus cerebrale, rappresentano la più frequente causa di morte nei pazienti affetti da IRC.

Solo due studi precedenti hanno valutato la prevalenza di LC in pazienti affetti da IRC (35-36). Il primo di questi ha utilizzato un ecografo B-mode studiando 50 pazienti in emodialisi. Le conclusioni furono che la presenza di LC in questi pazienti fosse dovuta all'ipertensione (35). Il secondo studio prese in esame 27 soggetti uremici. La metodica utilizzata fu l'analisi dell'onda doppler, tecnica che è riconosciuto essere poco accurata per la diagnosi di lesioni parietali iniziali non stenosanti. Le conclusioni di questo studio furono, comunque, simili al primo, sottolineando il fondamentale ruolo dell'ipertensione sullo sviluppo di LC (36).

Nella nostra ricerca abbiamo utilizzato un sistema duplex ad alta risoluzione, che consente la valutazione anche di lesioni parietali di piccole dimensioni come gli ispessimenti intimomediali. È stato esaminato un numero relativamente grande di pazienti con IRC non diabetica-non nefrosica ed un gruppo comparabile per numerosità e caratteristiche demografiche di soggetti di controllo.

La prevalenza di LC è risultata più elevata nei pazienti con IRC.

Solo tre pazienti presentavano stenosi gravi con indicazione chirurgica, mentre la maggior parte aveva stenosi di grado lieve o moderato.

Una probabile spiegazione di questa bassa frequenza di lesioni gravi può dipendere dall'avere escluso, dalla nostra casistica, i pazienti affetti da diabete mellito e/o quelli che riferivano sintomi e storia clinica positiva per patologia cerebrovascolare. La popolazione da noi esaminata rappresenta, quindi, probabilmente un campione selezionato rispetto alla popolazione generale di soggetti affetti da IRC afferente nei nostri ambulatori e/o reparti.

L'importanza clinica delle LC, comun- 
TABELLA III - CARATTERISTICHE DEI PAZIENTI NORMOTESI CON INSUFFICIENZA RENALE (GRUPPO I) E DEI NORMOTESI DI CONTROLLO (GRUPPO II)

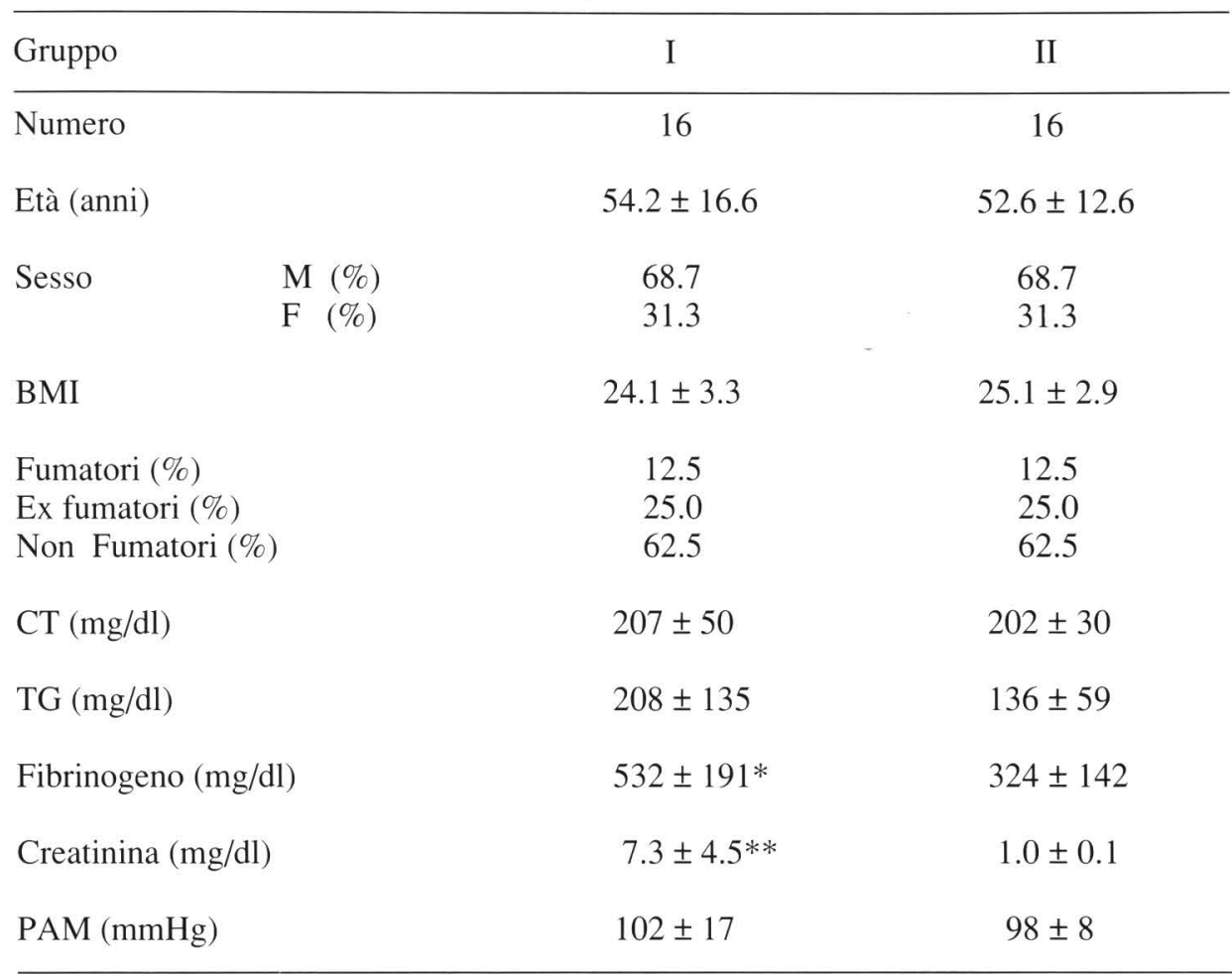

Valori con più e meno sono Media \pm Deviazione Standard

BMI: Indice di Massa Corporea; PAM: Pressione arteriosa media;

CT: Colesterolo Totale; TG: Trigliceridi.

$* \mathrm{p}<0.01$

$* * \mathrm{p}<0.0001$

que, non è limitata alle sole stenosi gravi per una serie di considerazioni:

1) Le placche aterosclerotiche possono produrre sintomi cerebrovascolari anche attraverso meccanismi diversi dal grado di stenosi, quali l'embolia o la trombosi su placca.

2) Le carotidi possono essere considerate una "finestra" sul sistema arterioso del paziente fornendo informazioni indirette anche sulla presenza di un coinvolgimento di altri distretti come l'arco aortico, le arterie intracraniche e le coronarie. 3) Le stenosi emodinamicamente non significative possono diventarlo, soprattutto se non si correggono i fattori di rischio per l'aterosclerosi.

Pertanto ciò che è emerso dal nostro studio sull'aumentata prevalenza di LC nei pazienti con IRC assume un interesse sia clinico che scientifico.

Abbiamo pensato che l'ipertensione potesse spiegare l'aumentata prevalenza di LC nei soggetti con IRC, come segnalato negli studi citati in precedenza $(35,36)$, TROLLO (GRUPPO II)

IMT $=$ Ispessimento intimo mediale $4=75-99 \%$; Classe $5=100 \%$.

$* \mathrm{p}=0.004$ ma sono emerse alcune evidenze contro tale ipotesi.

Nei due gruppi, infatti, non sono state trovate differenze significative per prevalenza, durata e trattamento dell'ipertensione, ma è stata trovata una differenza significativa nella prevalenza di LC. Inoltre, non è stata osservata una correlazione tra gravità delle lesioni carotidee e livelli pressori nei pazienti uremici.

La prevalenza di lesioni carotidee è risultata uguale negli ipertesi e nei normotesi del gruppo con IRC. Infine, confrontando tra loro i pazienti ipertesi e normotesi dei due gruppi è emersa una differenza significativa solo tra i normotesi (Fig. 2).

Da tutti gli elementi su riportati, quindi, sembra che l'ipertensione non sia il fattore discriminante per l'instaurarsi delle LC. L'IRC potrebbe, perciò, produrre un'accelerata aterosclerosi attraverso meccanismi diversi dall'ipertensione.

È necessaria, comunque, cautela nelle conclusioni, soprattutto considerando il piccolo numero di soggetti normotesi nei due gruppi. Si sa che nell'IRC solo una minoranza di soggetti è normotesa (7) $\mathrm{e}$ dei 103 soggetti della nostra casistica solo 16 erano normotesi $(15.6 \%)$.

Non possiamo escludere, inoltre, che un maggior carico emodinamico legato ad elevati livelli notturni di pressione nei pazienti uremici (37) e da noi non consi-

TABELLA IV - NUMERO DI ISPESSIMENTI MEDIO INTIMALI E PLACCHE ATEROSCLEROTICHE NEI PAZIENTI CON INSUFFICIENZA RENALE CRONICA (GRUPPO I) E NEI SOGGETTI DI CON-

\begin{tabular}{|c|c|c|}
\hline Gruppo & I & II \\
\hline Pazienti numero & 101 & 100 \\
\hline IMT & 37 & 34 \\
\hline \multicolumn{3}{|l|}{ Placche } \\
\hline Classe 1 & 26 & 19 \\
\hline Classe 2 & 36 & 20 \\
\hline Classe 3 & 4 & 2 \\
\hline Classe 4 & 3 & 0 \\
\hline Classe 5 & 0 & 0 \\
\hline Totale & 69 & 41 \\
\hline Lesioni totali $106^{*}$ & 75 & \\
\hline
\end{tabular}

Gravità delle stenosi: classe $1=1-19 \%$; Classe $2=20-49 \%$; Classe $3=50-74 \%$; Classe 


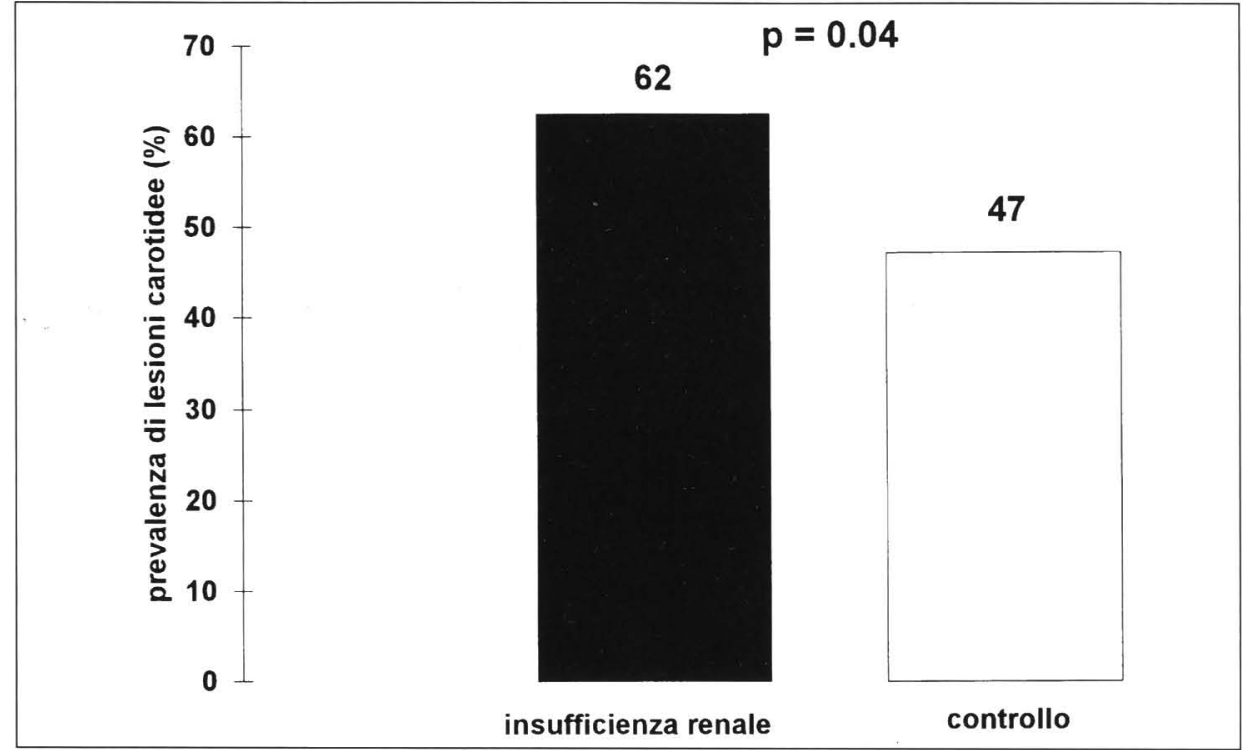

Fig. 1 - Prevalenza di lesioni carotidee nei pazienti con insufficienza renale cronica (istogramma nero) e nel gruppo di controllo (istogramma chiaro).

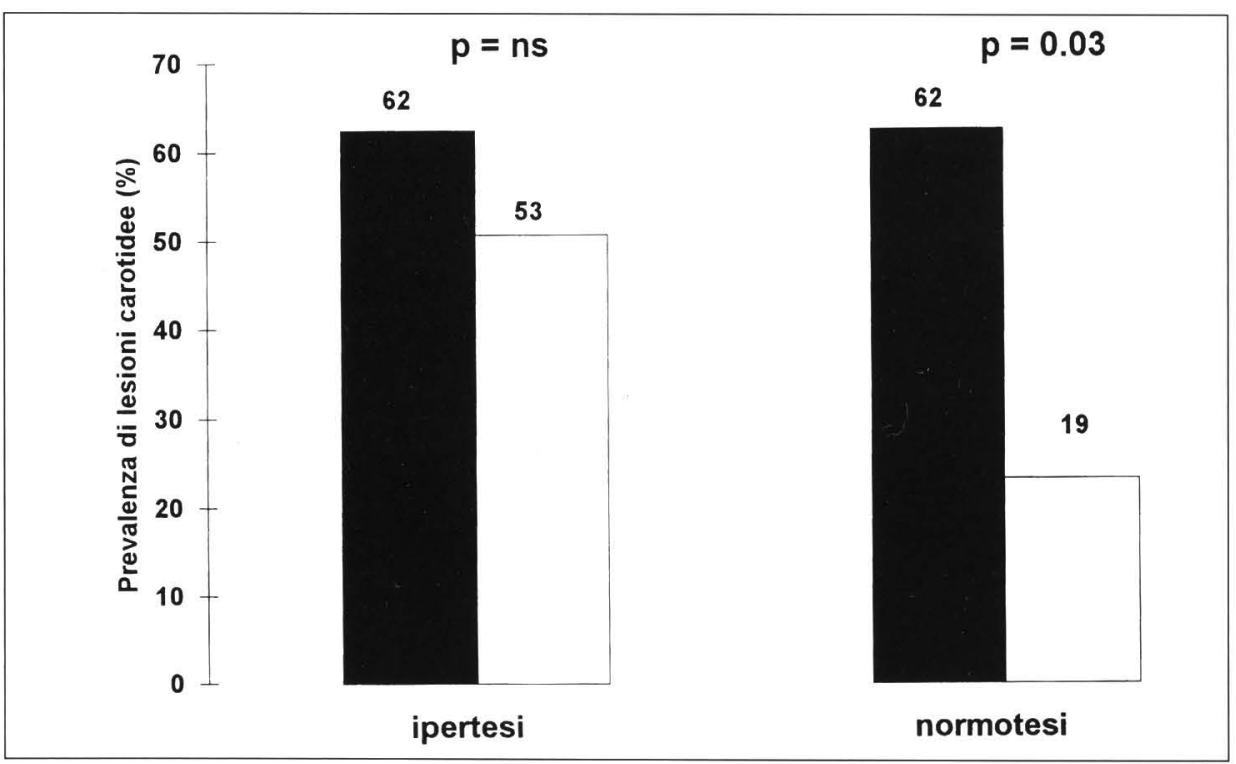

Fig. 2 - Prevalenza di lesioni carotidee nei pazienti con insufficienza renale cronica (istogrammi neri) e nel gruppo di controllo (istogrammi chiari) suddivisi in ipertesi e normotesi.

derato in questo studio, possa contribuire ad aumentare il danno vascolare nel gruppo di pazienti con IRC. Non si può negare, infine, che un efficace trattamento dell'ipertensione possa ridurre la progressione dell'aterosclerosi nei soggetti con insufficienza renale cronica (38).

Come segnalato nei risultati, si sono riscontrati un più basso BMI e più alti livelli di fibrinogeno e trigliceridi nei pazienti uremici rispetto al gruppo di controllo.
Questi dati meritano alcuni commenti. L' IRC induce una modificazione del regime dietetico e delle abitudini di vita ed una riduzione della massa muscolare, tutto ciò può spiegare valori più' bassi di BMI.

L'aumento del fibrinogeno nei pazienti uremici già descritto in studi precedenti $(6,15)$ è stato confermato dai nostri risultati. Va rilevato che il fibrinogeno è un importante fattore di rischio indipendente per l'aterosclerosi e per le complicanze che ne derivano quali l'infarto miocardico e l'ictus (39-43).

Pertanto, attualmente si raccomandano provvedimenti atti a ridurre il fibrinogeno, se elevato, nel tentativo di limitare l'incidenza di eventi ischemici vascolari (44).

Il fibrinogeno è risultato positivamente correlato con il grado di stenosi carotidee nei nostri pazienti uremici. Pertanto, i nostri dati sembrano suggerire l'utilità di determinare i livelli di fibrinogeno nei pazienti con IRC al momento della valutazione iniziale e nel follow-up.

Una correlazione significativa dell'indice di LC è stata osservata, inoltre, con età, numero di leucociti e livelli di trigliceridi. Non sono state rilevate correlazioni con gli altri parametri lipidici, comprese le lipoproteine e le frazioni apoproteiche.

L'età è un fattore di rischio indipendente per l'aterosclerosi, non modificabile e l'invecchiamento della popolazione uremica costituisce uno dei fattori più importanti per spiegare l'aumentata morbilità e mortalità cardiovascolare, evidenziata negli ultimi anni. Un numero elevato di leucociti periferici, è stato correlato con un aumento del rischio di infarto miocardico (45, 46). È noto, inoltre, che i leucociti partecipano al complesso meccanismo che comprende la promozione, lo sviluppo e le complicanze delle placche aterosclerotiche $(47,48)$.

Pertanto non sorprende il fatto di aver trovato una correlazione fra il numero di globuli bianchi e la gravità delle LC. Le lipoproteine ricche di trigliceridi sono considerate un importante fattore di rischio per l'aterosclerosi (49-51), l'ipertrigliceridemia è una delle alterazioni lipidiche più frequenti nei pazienti con IRC $(9,10,52)$ e sembra legata alla presenza di un ridotto metabolismo (7) tipico di questa patologia. Anche noi abbiamo riscontrato aumentati livelli di trigliceridi (Tab. I) tra i pazienti uremici. Elevati livelli di BVLDL, che sono lipoproteine ricche di trigliceridi altamente aterogene per l'alta affinità con i macrofagi, sono stati segnalati in soggetti affetti da IRC $(52,53)$.

Pertanto, come ci si poteva aspettare, anche nel nostro studio è stata trovata una significativa correlazione fra indice di LC e livelli sierici di trigliceridi. 


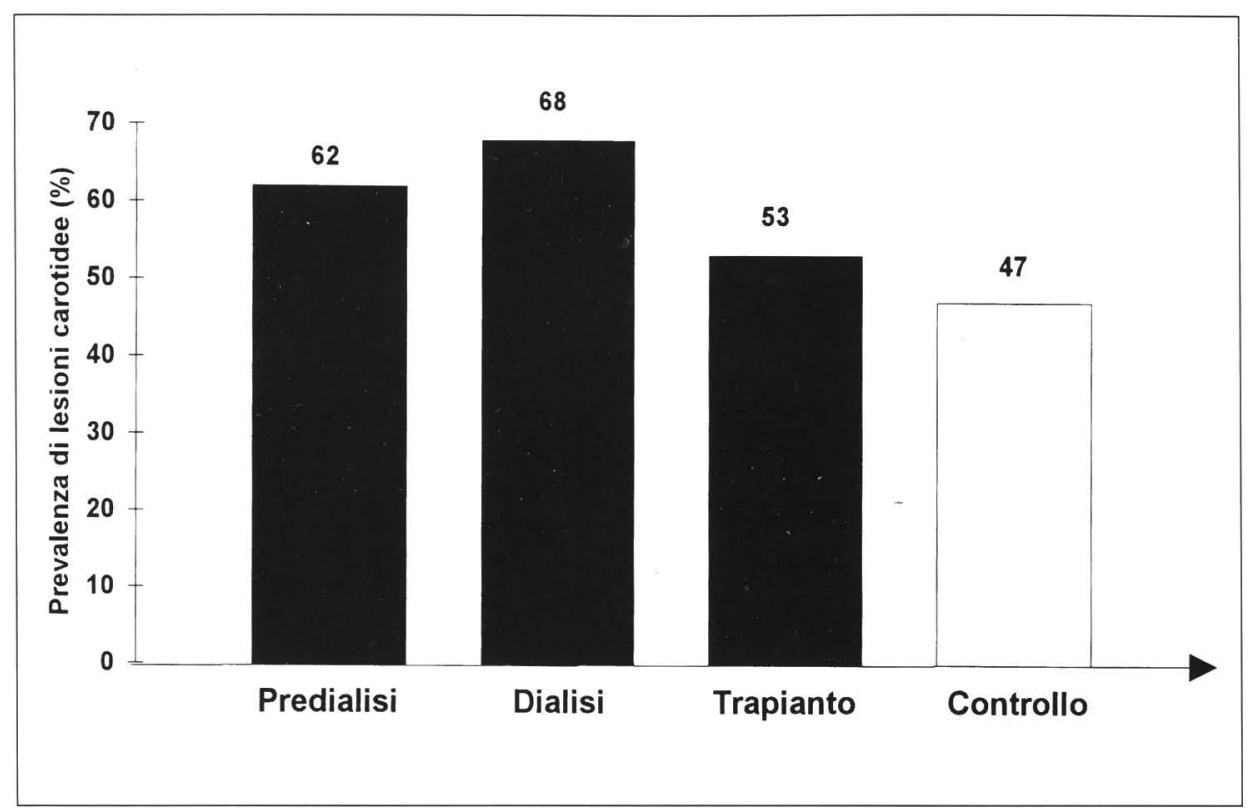

Fig. 3 - Prevalenza di lesioni carotidee nei tre sottogruppi di pazienti con insufficienza renale cronica (istogrammi neri) e nel gruppo di controllo (istogramma chiaro).

\section{Conclusioni}

I pazienti affetti da insufficienza renale cronica non su base diabetica, presentano un più importante interessamento aterosclerotico delle carotidi rispetto ad una popolazione di controllo con simili caratteristiche demografiche. La studio con metodi non invasivi quali il sistema duplex è, perciò, consigliabile nella valutazione iniziale e nel follow-up di questi soggetti anche se non sintomatici per cerebrovasculopatia.

La maggior prevalenza di lesioni aterosclerotiche nei pazienti con IRC non può essere correlata solo con l'aumento dei livelli pressori e, quindi, con l'alterazione di un singolo parametro.

Più fattori, infatti, al di fuori dell'ipertensione, quali alterazioni della coagulazione, dei leucociti ed aumento dei trigliceridi, potrebbero spiegare la maggior gravità di aterosclerosi nei pazienti uremici rispetto ai controlli.

La correlazione fra lesioni carotidee e livelli di fibrinogeno e trigliceridi nella popolazione con IRC suggerisce la possibilità di interventi preventivi e terapeutici atti a ridurre questi due fattori di rischio nella speranza di prevenire le complicanze ischemiche dell'aterosclerosi.

\section{Ringraziamento}

Gli Autori ricordano con riconoscenza il Professor Arturo Borsatti, Direttore della Cattedra di Nefrologia I dell'Università di Padova e Preside della Facoltà di Medicina e Chirurgia della stessa Università deceduto prematuramente durante lo svolgimento di questo lavoro. Il Suo contributo nella ideazione e supervisione di questa ricerca è stato fondamentale.

\section{BIBLIOGRAFIA}

1. Gurland HJ, Brunner FP, Dehn HV, Harlen H, Parsons FM, Scharer $\mathrm{K}$. Combined report on regular dialysis and transplantation in Europe. Proc Eur Dial Transplant Assoc 1973; 10: XVII-LVII.

2. Burton BT, Krueger KK, Bryan FA Jr: National Registry of long-term dialysis patients. JAMA * 218: 718-722; 1971.

3.

Degoulet P, Legrain M, Reach $\mathrm{I}$, et al. Mortality risk factors in patients treated by chronic hemodyalisis. Nephron 31: 103-110; 1982.

4. Lindner A, Charra B, Sherrard D, Scribner BH: Accelerated atherosclerosis in prolonged maintenance hemodialysis. N Engl J Med 290: 697-701; 1974.

5. Broyer M, Brunner FP, Brynger $\mathrm{H}$, et al. Combined report on regular dialysis and transplantation in Europe. Proc Eur Dial Transplant Assoc 19: 2-60; 1982.

6. Ibels LS, Stewart JH, Mahony JF, Neale FC, Sheil GR. Occlusive arterial disease in uraemic and hemodialysis patients and renal transplant recipients. Q J Med 44: 197214; 1977.

7. King WM, Greene EL, Raij L. Cardiovascular risk factors in chronic renal failure and hemodialysis population. Am J Kidney Dis 19: 505-13, 1992.

8. Zucchelli P, Santoro A, Zuccala A. Genesis and control of hypertension in hemodialysis patients. Semin Nephrol 8: 163-8; 1988.

9. Appel G. Lipid abnormalities in renal disease. Kidney Int 39: 169$83 ; 1991$.

10. Cramp DG. Plasma lipid alterations in patients with chronic renal disease. CRC Crit Rev Clin Lab Sci 17: 77-101; 1982.

11. Parra HJ, Mezdour H, Cachera 
C, Dracon M, Tacquet A, Fruchart JC. $L p($ a) lipoprotein in patients with chronic renal failure treated by hemodialysis. Clin Chem 1987; 33: 721-1.

12. Attman P, Alaupovic P, Gustavson A. Serum apolipoprotein profile of patients with chronic renal failure. Kidney Int 1987; 32: 368-75.

13. Grutzmacher P, Marz W, Peschke B, Gross W, Schoeppe W. Lipoproteins and apolipoproteins during the progression of chronic renal disease. Nephron 1988; 50: 103-11.

14. Attman P, Alaupovic P. Lipid and apolipoprotein profiles of uremic dyslipoproteinemia-Relation to renal function and dialysis. Nephron 1991; 57: 401-10.

15. Jubiliber SJ. Hemostatic abnormalities in renal disease. Am J Kidney Dis 1985; 5: 219-25.

16. Warwick GL, Packard CJ. Lipoprotein metabolism in the nephrotic syndrome. Nephrol Dial Transplant 1993; 8: 385-96.

17. Radhakrishnan J, Appel AS, Valeri A, Appel GB. The nephrotic syndrome, lipids, and risk factors for cardiovascular disease. Am J Kidney Dis 1993; 22: 135-42.

18. Tell GS, Howard G, Mc Kinney WM, Toole JF. Cigarette smoking cessation and extracranial carotid atherosclerosis. JAMA 1989; 261: 1178-80.

19. Burstein M, Scholnick HR, Morgin R. Rapid method for the isolation of lipoproteins from human serum by precipitation with polyanions. J Lipid Res 1970; 11: 583-95.

20. Friedwald WT, Levy RI, Fredrickson DS. Estimation of plasma low density lipoproteins cholesterol concentration without use of the preparative ultracentrifuge. Clin Chem 1972; 18: 499-509.

21. Fless GM, Snyder ML, Scanu AM. Enzyme-linked immunoassay for Lp(a). J Lipid Res 1989; 30: 651-61.
22. Felix WR jr. Duplex ultrasound scanning for carotid artery disease. In: noninvasive diagnosis of peripheral vascular disease. New York, NY: Raven Press, 1988; 107.

23. Pignoli P, Tremoli E, Poli A, Oreste PL, Paoletti R. Intimal plus medial thickness of the arterial wall: a direct measurement with ultrasound imaging. Circulation 1986; 74: 1399-406.

24. Rossi GP, Rossi A, Zanin L, et al. Excess prevalence of extracranial carotid artery lesions in renovascular hypertension. Am J Hypertension 1992; 5: 8-15.

25. Rossi GP, Rossi A, Zanin L, Calabrò A, Crepaldi G, Pessina AC. Prevalence of extracranial carotid artery lesions at duplex in primary aldosteronism. Am J Hypertension 1993; 6: 8-14.

26. Salonen R, Seppanen K, Raurama R, Salonen JK. Prevalence of carotid atherosclerosis and serum cholesterol levels in Eastern Finland. Arteriosclerosis 1988; 8: 788-92.

27. Tell GS, Howard G, McKinley WM. Risk factors for site specific extracranial carotid artery plaque distribution as measured by B-mode ultrasound. J Clin Epidemiol 1989; 42: 551-59.

28. Hennerici M, Reifschneider G, Trockel U, Aulich A. Detection of early atherosclerotic lesions by duplex scanning of the carotid artery. J Clin Ultrasound 1984; 12: 455-64.

29. Poli A, Tremoli E, Colombo A, Sirtori M, Pignoli P, Paoletti R. Ultrasonographic measurement of the common carotid artery wall thickness in hypercholesterolemic patients. A new model for the quantitation and follow-up of preclinical atherosclerosis in living human subjects. Atherosclerosis 1988; 70: 253-61.

30. Roussel L, Steyaert L, Meeus L, Vandevoorde P, Devos V, Patyn G. Duplex sonography of the cervical carotid artery. Part II: Confrontation with angiography. Criteria for flow restrictive stenoses. JBR-BTR 1988; 71: 589-600.

31. Cranley JJ Jr, Carcow WS, Baldridge ED. Atlante di Duplex scanner. Torino, Italy: Centro Scientifico Editore, 1990; 41-83.

32. Rosner B. Fundamentals of Biostatistics. Boston, Massachusetts: Duxbury Press, 1986; 333-68.

33. Godfrey K. Comparing the means of several groups. In: Bailar III JC, Mosteller F eds. Medical Uses of Statistics. Boston, Massachusetts: NEJM Books, 1992; 233-57.

34. Kleinbaum DG, Kupper LL, Muller KE. Applied regression analysis and other multivariable methods. Boston, Massachusetts: PWS Kent Publ. Co, 1988; 102-114.

35. Neveling M, Hoier C, Bewermeyer H, Mertens M. B scan ultrasound findings in the carotid bifurcation area of hemodialysis patients. Ultraschall Med 1992; 13: 127-31.

36. Bernardi D, Ferreri A, Moretti P, Nesti E, Urti DA, Bonechi I. Carotid artery atherosclerotic disease assessed by flow velocity wave form analysis in hemodialyzed normotensive and hypertensive patients. Nephron 1986; 44: 180-5.

37. Baumgart $\mathrm{P}$, Walger $\mathrm{P}$, Gerke M, Dorst KG, Vetter H, Rahn KH: Nocturnal hypertension in renal failure, haemodialysis and after renal transplantation. J Hypertens 1989; 7 (Suppl): S70-1.

38. Vincenti F, Amend W, Abele J, Feduska NJ, Salvatierra O. The role of hypertension in hemodialysis-associated atherosclerosis. Am J Med 1980; 68: 363-9.

39. Wilhelmsen L, Svardsudd K, Korsan-Bengtsen K, Larsson B, Welin L, Tibblin G. Fibrinogen as a risk factor for stroke and myocardial infarction. N Engl J Med 1984; 311: 501-5.

40. Meade TW, Brozovic M, Chakrabarti RR, et al. Haemostatic function and ischaemic heart disease: principal results of the 
Northwick Park Heart Study. Lancet 1986; 2: 533-7.

41. Cook NS, Ubben D. Fibrinogen as a major risk factor in cardiovascular disease. Trends Pharmacol Sci 1990; 11: 444-51.

42. Moller L, Kristensen TG. Plasma fibrinogen and ischemic heart disease risk factor. Arterioscl Thromb 1991; 11: 344-50.

43. Qizilbash N, Jones L, Warlow C, Mann J. Fibrinogen and lipid concentration as risk factors for transient ischaemic attacks and minor ischaemic strokes. Br Med J 1991; 303: 605-9.

44. Clark WM, Coull BM, Beamer NB. Need for treatment of elevated plasma fibrinogen levels in cerebrovascular disease. Heart Disease and Stroke 1993; 2: 503-6.

45. Yarnell JWG, Baker IA, Sweetnam PM, et al. Fibrinogen, viscosity, and white blood cell count are major risk factors for ischemic heart disease. The Caerphilly and Spedwell collaborative heart studies. Circulation 1991; 83: 836-44.

46. Ensrud K, Grimm RH Jr. The white blood cell count and risk for coronary heart disease. Am Heart J 1992; 124: 207-13.

47. Ross R. The pathogenesis of atherosclerosis: a perspective for the 1990s. Nature 1993; 362: 801-9.

48. Jackson MH, Collier A, Nicoli JJ, et al. Neutrophil count and activation in vascular disease. Scott Med J 1992; 37: 41-3.

49. Tornvall P, Bavenholm P Landou C, De Faire U, Hamsten A. Relation of plasma levels and composition of apolipoprotein B-containing lipoproteins to angiographically defined coronary artery disease in young patients with myocardial infarction. Circulation 1993; 88: 2180-9.

50. Castelli WP. Epidemiology of triglycerides: a view from Framingham. Am J Cardiol 1992; 70: 3H$9 \mathrm{H}$.
51. Gotto AM. Hypertrygliceridemia: risks and perspectives. Am J Cardiol 1992; 70: 19H-25H.

52. Cheung AK, Wu LL, Kablitz C, Leypoldt JK. Atherogenic lipids and lipoproteins in hemodialysis patients. Am J Kidney Dis 1993; 22: 271-6.

53. Steinber D, Parthasarathy S, Care TE, Khoo JC, Witztum JL. Beyond cholesterol: modifications of low-density lipoprotein that increase its atherogenicity. N Engl J Med 1989; 320: 915-24. 